\title{
Nuevas evidencias acerca de la presencia de Stegomastodon platensis Ameghino, 1888, Proboscidea: Gomphotheriidae, en el Pleistoceno tardío de Chile central
}

\section{New evidences on the presence of Stegomastodon platensis Ameghino, 1888, Proboscidea: Gomphotheriidae, in the Late Pleistocene of Central Chile}

\author{
R. Labarca ${ }^{1}$, M.T. Alberdi ${ }^{2}$, J.L. Prado ${ }^{3}$, P. Mansilla ${ }^{4}$, F.A. Mourgues ${ }^{5}$ \\ 1 LABPALEO, Instituto de Ciencias Ambientales y Evolutivas, Universidad Austral de Chile, Casilla 567, Valdivia, Chile. \\ Email: r.labarca.e@gmail.com \\ 2 Departamento de Paleobiología, Museo Nacional de Ciencias Naturales (CSIC), José Gutiérrez Abascal 2, 28006 Madrid, \\ España \\ 3 INCUAPA, Departamento de Arqueología, Universidad Nacional del Centro, Del Valle 5737, Olavarría, Argentina \\ 4 Museo de Historia Natural y Cultural del Desierto de Atacama, Av. Bernardo O’higgins s/n Interior del Parque El Loa, \\ Calama, Chile \\ 5 TERRA IGNOTA, Consultoría en Patrimonio y Geociencias. Dr. Càdiz 726, Santiago, Chile
}

\section{RESUMEN}

El presente trabajo contextualiza, describe, y asigna taxonómicamente los materiales craneales y dentales de Gomphotheriidae provenientes del sitio El Trebal 1, Pleistoceno tardío de Chile central. A partir de variables métricas y morfológicas los fósiles son incluidos en Stegomastodon platensis. Se discuten las implicancias taxonómicas de este hallazgo así como algunos antecedentes paleoambientales regionales.

Palabras clave: Gomphotheriidae; Stegomastodon platensis; Chile; Pleistoceno final.

\section{ABSTRACT}

This paper contextualizes, describes and taxonomically assigned cranial and dental Gomphotheriidae materials from the El Trebal 1, late Pleistocene form central Chile. Starting from metric and morphological variables fossils are included in Stegomastodon platensis. Taxonomic implications of this finding as well as some regional paleoenvironmental history are discussed.

Keywords: Gomphotheriidae; Stegomastodon platensis; Chile; Late Pleistocene dx.doi.org/10.3989/egeol.42199.385.

Copyright: ( 2016 CSIC. This is an open-access article distributed under the terms of the Creative Commons Attribution-Non Commercial (by-nc) Spain 3.0 License. 


\section{Introducción}

En Sudamérica, el orden Proboscídea se encuentra representado exclusivamente por una sola familia, Gomphotheriidae, la que aparentemente habría ingresado al subcontinente posterior al establecimiento del istmo de Panamá, en torno a los 2,5 Ma a juzgar por los registros más tempranos documentados (Reguero et al., 2007). Restos fósiles de gonfoterios asignados al Mioceno tardío (Campbell et al., 2000) han sido descartados debido a inconsistencias estratigráficas y de datación (Alberdi et al., 2004; Lucas, 2013; Mothé \& Avilla, 2015). Las formas que colonizan Sudamérica provienen de un stock ancestral norteamericano que se diversificó rápidamente durante el Mioceno medio tras su arribo desde Asia, hace unos 16 millones de años (Prothero et al., 2008).

La taxonomía de la familia en Sudamérica se encuentra aún en debate. Inicialmente, Alberdi y colaboradores (Alberdi et al., 2002, 2004; Prado et al., 2005) señalaron la presencia de dos géneros y tres especies: Cuvieronius hyodon Fisher 1814, Stegomastodon platensis Ameghino 1888 y Stegomastodon waringi Holland 1920. Alternativamente, Mothé et al. (2012, 2013; Mothé \& Avilla, 2015) y Lucas (2013, Lucas et al., 2011), entre otros, si bien concuerdan en señalar la presencia de $C$. hyodon, sugieren que debe utilizarse el nombre genérico Stegomastodon para las especies norteamericanas. En consecuencia, Mothé et al. (2012) revalidan el nombre genérico de Notiomastodon para todas formas de América del Sur hasta ahora incluidas en Stegomastodon y/o Haplomastodon. Asimismo, Mothé \& Avilla (2015) han propuesto incluir $S$. waringi en $N$. platensis $(=S$. platensis $)$ debido a las escasas diferencias entre ambas especies. Alberdi y colaboradores han coincidido con este último arreglo, considerando que las diferencias morfológicas entre ambas formas tendrían que mantenerse a nivel subespecífico (Recabarren et al., 2014).

En Chile, Frassinetti \& Alberdi (2000) señalaron la presencia exclusiva de Cuvieronius hyodon en todo el territorio, pero más tarde (Frassinetti \& Alberdi, 2005) indicaron la existencia de materiales asignables a Stegomastodon. Lo anterior, fue considerado por diversos autores como una eventual coexistencia de ambos géneros en Chile (p.e. Mothé et al., 2012, 2013; Lucas, 2013). Sin embargo, Labarca \& Alberdi (2011) y recientemente Recabarren et al. (2014) han argumentado que por el momento no existe evidencia sólida que sustente la presencia de Cuvieronius en Chile, ya que todos los materiales disponibles que presentan rasgos diagnósticos (p.e. defensas) son compatibles con el género Stegomastodon (= Notiomastodon) (contra Casamiquela 1999). Cronológicamente, todos los materiales Chilenos han sido asignados al Pleistoceno Superior (Casamiquela, 1999; Frassinetti \& Alberdi, 2000).

En este trabajo damos a conocer un nuevo registro fósil de gonfoterio proveniente de la localidad El Trebal 1 (Región Metropolitana, Chile), correspondiente a la porción anterior de un cráneo con sus respectivos molares y defensas. Los restos fueron descubiertos accidentalmente mientras se realizaban obras relacionadas con el tratamiento de aguas servidas de la ciudad de Santiago. Los materiales son estudiados sistemáticamente tanto desde una perspectiva morfológica como métrica. Para esto último se utilizaron datos existentes de otras localidades de América del Sur (Alberdi et al., 2002, 2004, 2008; Labarca \& Alberdi, 2011; Prado \& Alberdi, 2012; Prado et al., 2002, 2005). Se discuten las implicancias taxonómicas de este hallazgo así como algunos antecedentes paleoambientales regionales.

\section{Marco estratigráfico y cronológico}

El sitio El Trebal 1 se ubica en la depresión intermedia de Chile central (Figura 1), específicamente en la hoya hidrográfica del río Mapocho, en la cuenca de Santiago. El relleno de la cuenca de Santiago está formado por un conjunto de unidades geológico geomorfológicas correspondiente a depósitos de cenizas ignimbríticas pumicíticas, abanicos aluvionales, depósitos fluviales, coluviales y conos de deyección, depósitos lacustres y depósitos de remoción en masa (Varela, 1991). La cuenca de Santiago recibe aportes sedimentarios de los ríos Maipo y Mapocho, y de los esteros Lampa y Angostura. Los materiales paleontológicos provienen del sector suroccidental de la cuenca, al sur de los cerros de la Puntilla del Viento, de los depósitos fluviales aterrazados antiguos del río Mapocho, atribuidos al Pleistoceno Superior Holoceno (Sellés \& Gana, 1991; Figura 2). 


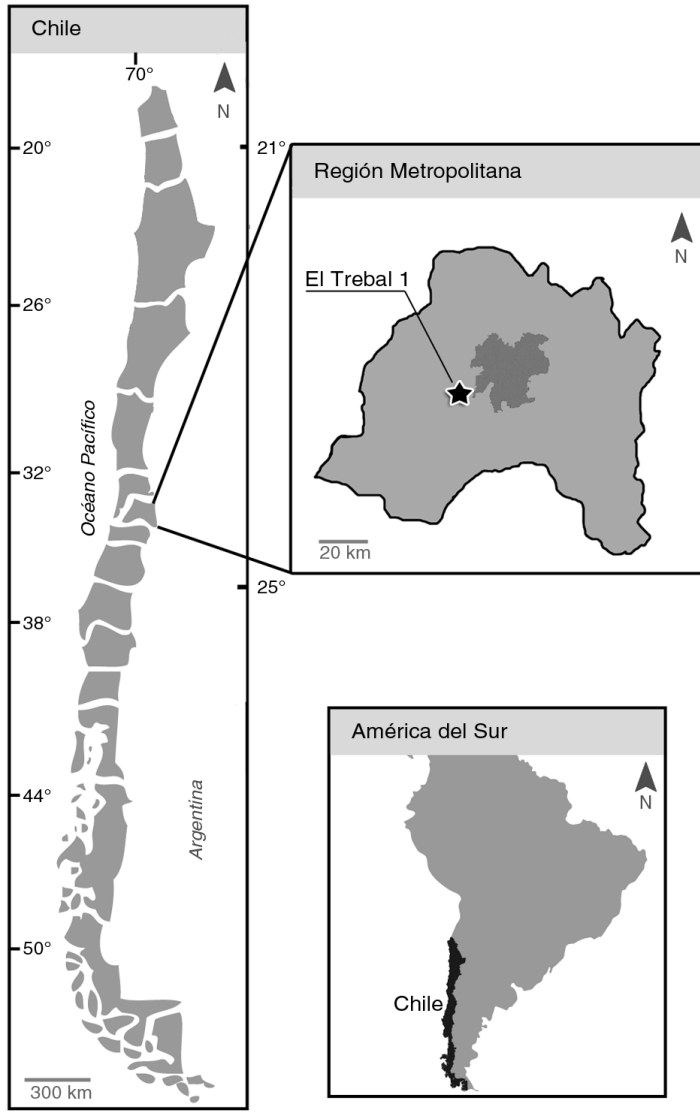

Figura 1.-Ubicación del sitio El Trebal 1.
El perfil estratigráfico levantado en el lugar del hallazgo (ET1) permitió documentar una sucesión de al menos diez estratos conformados fundamentalmente por conglomerados de clastos de distintos tamaños y arenas finas. Se trata de depósitos fluvio-aluviales del río Mapocho, los que rellenaron el paleo relieve de la cuenca de Santiago durante el Pleistoceno tardío - Holoceno temprano, con una dirección de relleno hacia el norte (Figura 3). Específicamente, los restos de gonfoterios provienen de la capa ET1-10 compuesta por un conglomerado de clasto soportado, selección moderada a mala, con bolsones limo arcillosos (Figura 3).

\section{Materiales y métodos}

El material fósil fue recuperado mediante una excavación sistemática de once unidades de $1 \mathrm{~m}^{2}$ nombradas alfanuméricamente. Cada unidad fue intervenida con niveles artificiales de $10 \mathrm{~cm}$ hasta una profundidad variable de entre 10 y $75 \mathrm{~cm}$, considerando como nivel 0 aquel dejado por la maquinaria que descubrió los restos (Figura 4). Los sedimentos fueron cernidos con malla de $0,4 \mathrm{~mm}$ con el objeto de recuperar restos fragmentados u otras evidencias contextuales (restos vegetales, microfauna, etc.).

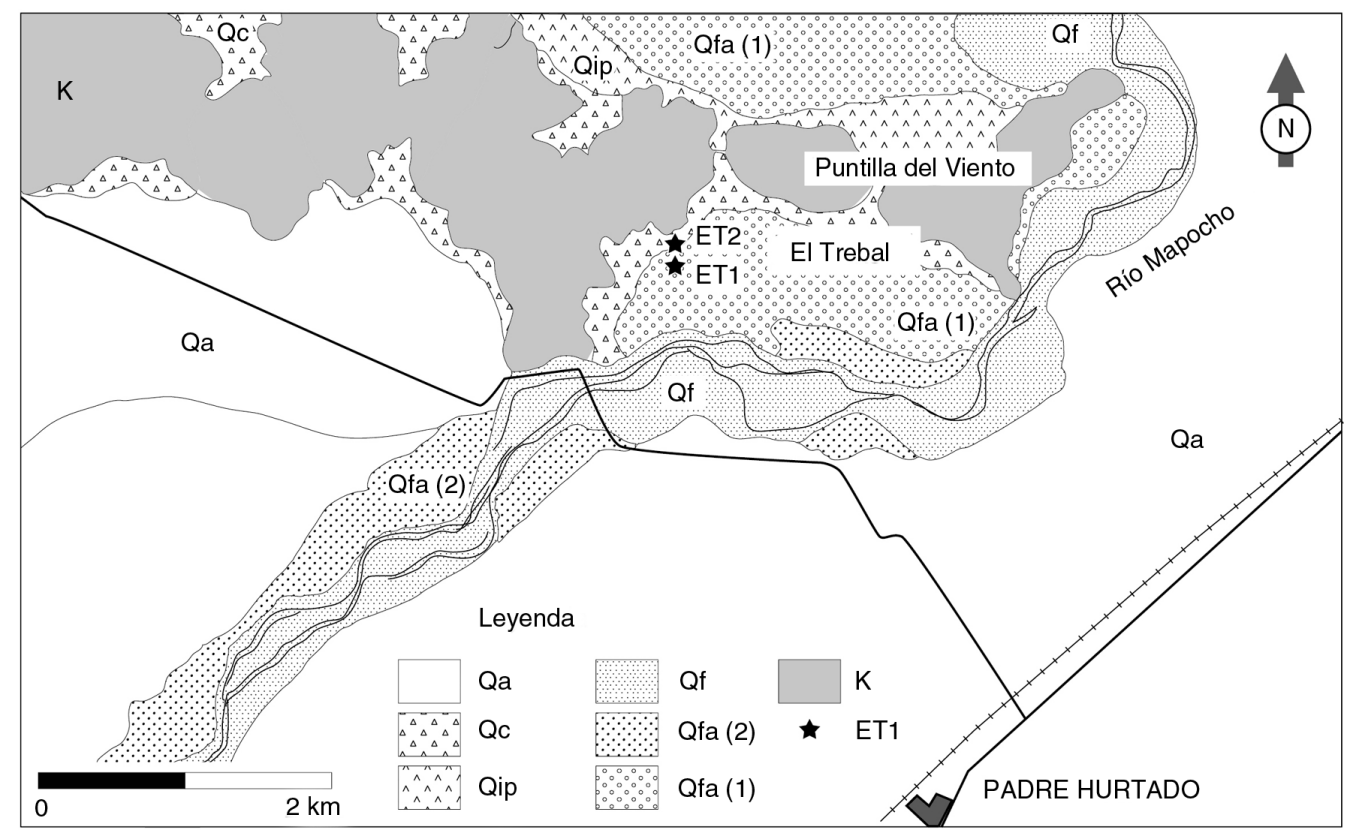

Figura 2.-Detalle del emplazamiento del sitio El Trebal 1 (modificado de Sellés \& Gana, 1991). Q: Pleistoceno-Holoceno, a: Aluvial; c: coluvial; f: fluvial; ip: volcánico piroclástico; K: Cretácico. 
Sitio El Trebal 1

(ET1)

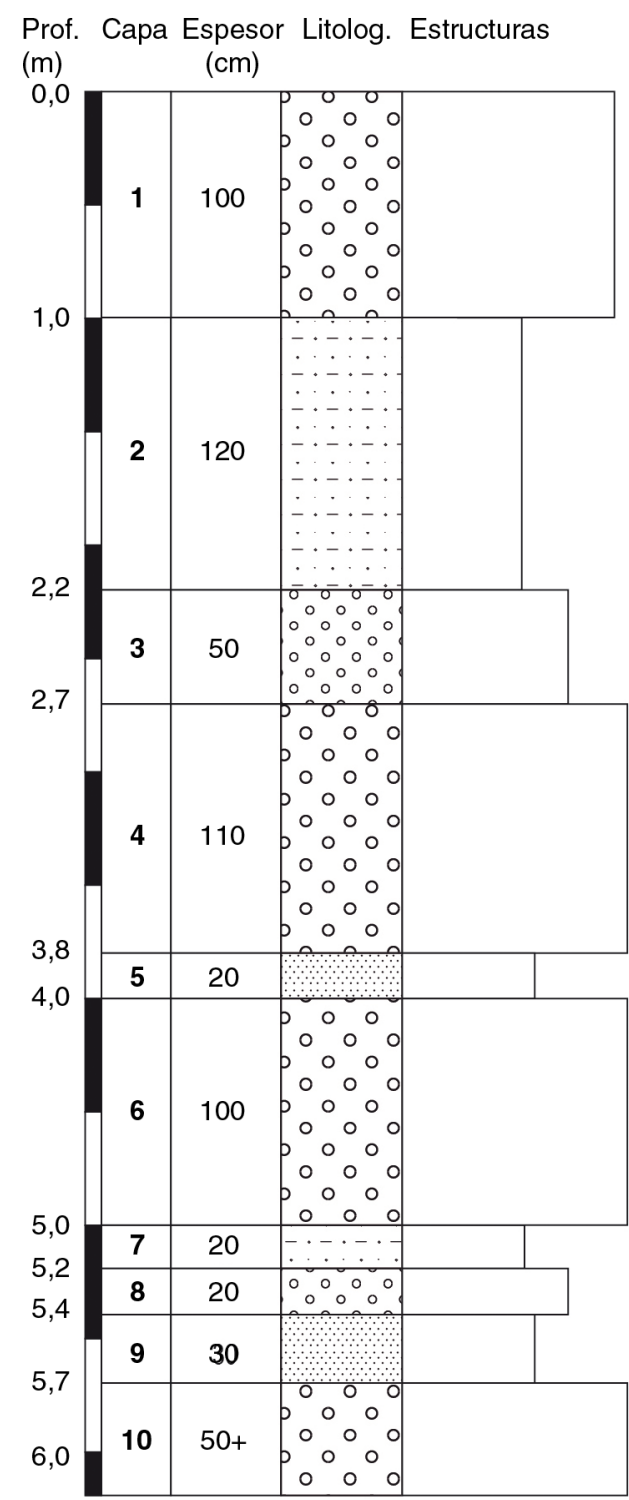

Ambiente

Fluvial LEYENDA

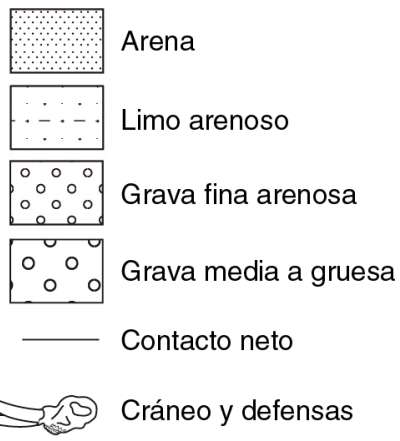

Figura 3.-Columna estratigráfica el sitio El Trebal 1.

El material en estudio fue comparado morfológica y biométricamente con ejemplares procedentes de varias localidades de América del Sur depositados en distintos museos e instituciones (Alberdi et al., 2002, 2004, 2008; Labarca \& Alberdi, 2011; Prado \& Alberdi, 2012; Prado et al., 2002, 2005; Recabarren et al., 2014). Para la descripción y el análisis morfométrico de los restos molarifornes y craneales se tomaron medidas de acuerdo Alberdi et al. (2002; Figura 5). De los restos dentarios se tomaron las siguientes dimensiones: longitud total (L), anchuras máximas a nivel de cada colina o lofos/lófidos (A1 a A5), y anchura del talón (T). De los restos craneales se seleccionaron las siguientes medidas: 6. Anchura máxima sub-orbitaria; 7. Anchura de los premaxilares al nivel del orificio infraorbitario; 10. Anchura entre 1os pseudomeatos auditivos externos; 11. Anchura entre 1os bordes externos de las fosas de articulación mandibulares; 12. Anchura entre los bordes internos de las fosas de articulación mandibular; 13. Anchura 


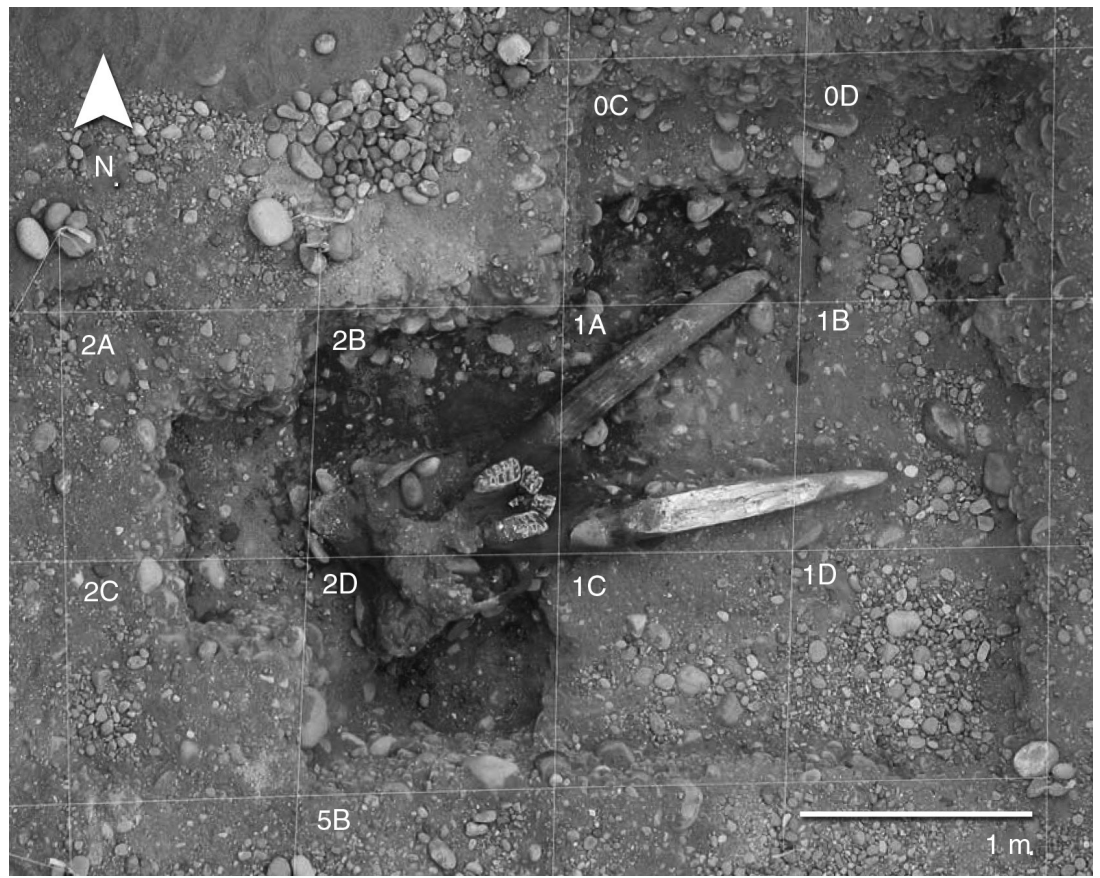

Figura 4.-Planta de la excavación del gonfoterio de El Trebal 1.

A.

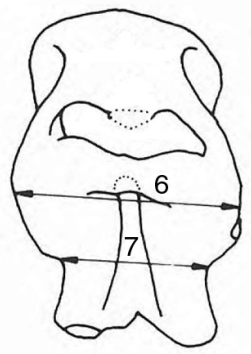

D.

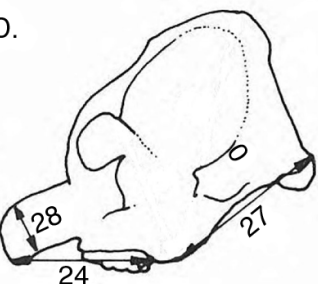

B.

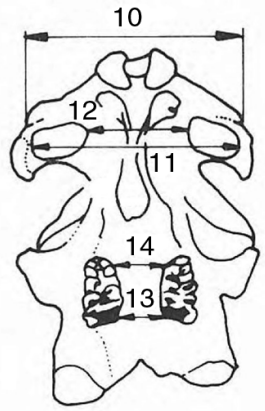

E.

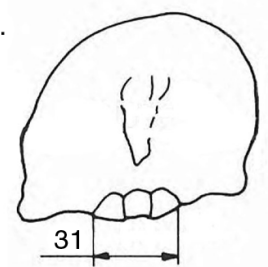

C.

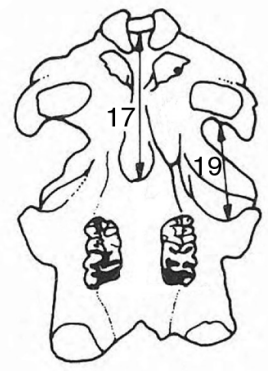

F.

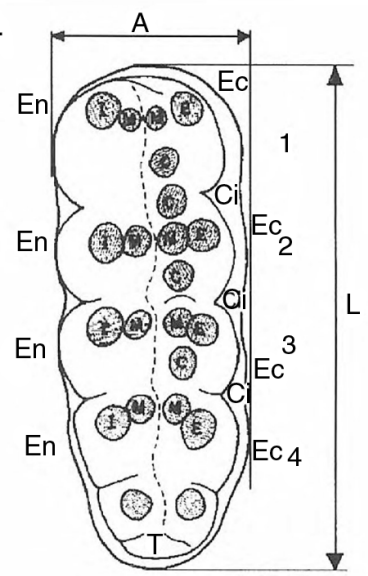

Figura 5.-Medidas utilizadas en el presente estudio. Cráneo: A. vista dorsal; B. y C. vista ventral; D. vista lateral; E. vista posterior. Molar: F. vista oclusal (Modificado de Alberdi et al., 2002). Explicación de los números y abreviaturas en el texto. 
entre 1 os bordes antero-interno de 1os molares; 14. Anchura entre 1os bordes postero-interno de 1 os molares; 17. Distancia entre la espina palatina y el borde anterior del foramen magnum; 19. Diámetro máximo antero-posterior de la fosa temporal a nivel de la arcada yugal; 24 . Distancia entre el borde antero-inferior del premaxilar y la apófisis pterigoidea; 27. Distancia entre el borde posterior de 1os cóndilos occipitales y la apófisis pterigoidea; 28. Diámetro máximo antero-posterior de 1os premaxilares; 31. Anchura entre 1os bordes latero-externos de los cóndilos occipitales; 32. Diámetro máximo del agujero magnum (no figurado).

Los datos obtenidos se compararon con otros restos previamente estudiados por Alberdi \& Prado (Alberdi et al., 2002, 2004, 2008; Labarca \& Alberdi, 2011; Prado \& Alberdi, 2012; Prado et al., 2002, 2005; Recabarren et al., 2014) de otras localidades de América del Sur siguiendo la metodología de Alberdi et al. (2002). Con los restos dentarios se han realizado análisis bivariantes para los M2 y M3. Asimismo, se ha aplicado el índice de robustez de Osborn (1936) para los molares existentes (A máxima $\times 100 / L)$.

\section{Paleontología sistemática}

Orden Proboscidea Illiger, 1811

Familia Gomphotheriidae Cabrera, 1929

Género Stegomastodon Pohlig, 1912

Stegomastodon platensis Ameghino, 1888

Figura 6; Tabla 1 y 2

Material referido. Porción anterior de un cráneo con los dos M2-M3 derechos e izquierdos, y las dos defensas superiores completas, la derecha bien conservada (SGO.PV.19501), depositados en el Museo Nacional de Historia Natural de Santiago de Chile.
Descripción. El cráneo, de gran tamaño, pertenece a un individuo adulto con los M2 y M3 completos con desgaste avanzado, más avanzado en M2 que en M3, y ambas defensas. Originalmente completo in situ, la parte posterior se recuperó muy fragmentada por lo que sólo pudo ser registrada someramente en terreno previo a su extracción. Por tanto, la descripción que se presenta proviene fundamentalmente de la sección anterior del cráneo. En vista dorsal el espécimen SGO-PV.19501 retiene el hueso premaxilar y maxilar, pudiendo apreciarse el inicio del proceso zigomático de ambos maxilares y la porción anterior de la fosa subnasal. Esta última es marcadamente profunda y se diferencia claramente de la fosa incisiva, ubicada inmediatamente hacia la parte anterior, la que se encuentra poco desarrollada. Los procesos alveolares del premaxilar son robustos. Pese a estar parcialmente fracturada, es posible advertir que la incisura dental separa marcadamente ambas porciones anteriores de los premaxilares. Los alveolos de las defensas divergen levemente.

En vista ventral destaca el palatino, largo y angosto, con dos surcos a cada lado que rematan hacia anterior y posterior en dos agujeros bien delimitados. El maxilar está bastante hundido con los M2, bien desgastados, con tres lofos y casi en contacto en su parte mesio-lingual, los que además presentan un desgaste más acentuado en su superficie oclusal. El M3 derecho está entero, posee cuatro lofos y un talón fuerte o quinto lofo y un talón reducido; mientras que el izquierdo aunque tiene completo el alveolo, presenta la superficie oclusal totalmente alterada posdepositacionalmente a partir de la tercera colina en adelante, sólo observándose las dos primeras colinas bastante desgastadas. El desgaste mayor es lingual y los conos internos son más complicados que los externos. En los M2 queda perdida la línea media, mientras que en los M3 se conserva bien marcada, más clara en el M3 derecho. La morfología de los molares es bastante sencilla dado el grado

Tabla 1.-Medidas de molares $(\mathrm{mm})$ de Stegomastodon platensis de El Trebal 1

\begin{tabular}{|c|c|c|c|c|c|c|c|c|c|}
\hline $\begin{array}{l}\text { Numero de } \\
\text { colección }\end{array}$ & Molar & $\begin{array}{l}\text { Largo } \\
\text { total }\end{array}$ & $\begin{array}{l}\text { Ancho } \\
\text { lofo } 1\end{array}$ & $\begin{array}{l}\text { Ancho } \\
\text { lofo } 2\end{array}$ & $\begin{array}{l}\text { Ancho } \\
\text { lofo } 3\end{array}$ & $\begin{array}{l}\text { Ancho } \\
\text { lofo } 4\end{array}$ & $\begin{array}{l}\text { Ancho } \\
\text { talón }\end{array}$ & $\begin{array}{c}\text { Anchura } \\
\text { mayor }\end{array}$ & $\begin{array}{c}\text { Índice de } \\
\text { Osborn }\end{array}$ \\
\hline SGO.PV.19501 & M2 izquierdo & 153 & 94 & 92,5 & 83 & - & - & 94 & 61,44 \\
\hline SGO.PV.19501 & M2 derecho & 151 & 97,5 & 95,3 & 84,8 & - & - & 97,5 & 64,57 \\
\hline SGO.PV.19501 & M3 izquierdo & $225 \mathrm{ca}$ & 105 & 102,5 & 97 & - & - & 105 & 46,67 \\
\hline SGO.PV.19501 & M3 derecho & 225 & 102 & 100 & 100 & 88,5 & 67 & 102 & 45,33 \\
\hline
\end{tabular}


de desgaste, con los valles prácticamente cerrados y las figuras treboladas no muy complicadas y algo de rizo en el borde del esmalte. No se observan restos de cíngulo posiblemente debido al desgaste avanzado (Figura 6). En la parte mesial, los premaxilares, algo alterados, se encuentran soldados sin presentar divergencia en su zona mesial, sin embargo los alvéolos de las defensas están algo divergentes donde están alojadas las defensas, la izquierda algo alterada. Éstas son robustas y casi rectas con una ligera curvatura hacia arriba en el ápice. La defensa derecha, que es la más completa, alcanza una longitud de $1,2 \mathrm{~m}$, con una sección ligeramente ovalada cuyos diámetros en la base son $194 \times 144 \mathrm{~mm}$, a nivel medio de la longitud es de $183 \times 130 \mathrm{~mm}$ y en el ápice $106 \times 102,5 \mathrm{~mm}$. La defensa izquierda es similar en longitud pero está alterada en su parte más central. No se observan rastros de esmalte ni torsión en ninguna de ellas.

En vista lateral se advierte el hueso maxilar y premaxilar. En el primero destaca con claridad el agujero infraorbital, que es profundo y marcadamente ovalado; asociado a éste hacia lateral, es posible apreciar el proceso infraorbital del maxilar. Originalmente, la parte posterior del cráneo retenía el seno nucal, además de presentar cóndilos occipitales robustos.

Las dimensiones de los molares se entregan en la Tabla 1 (en mm), mientras que las medidas obtenidas para el cráneo se presentan en la Tabla 2 (en mm).

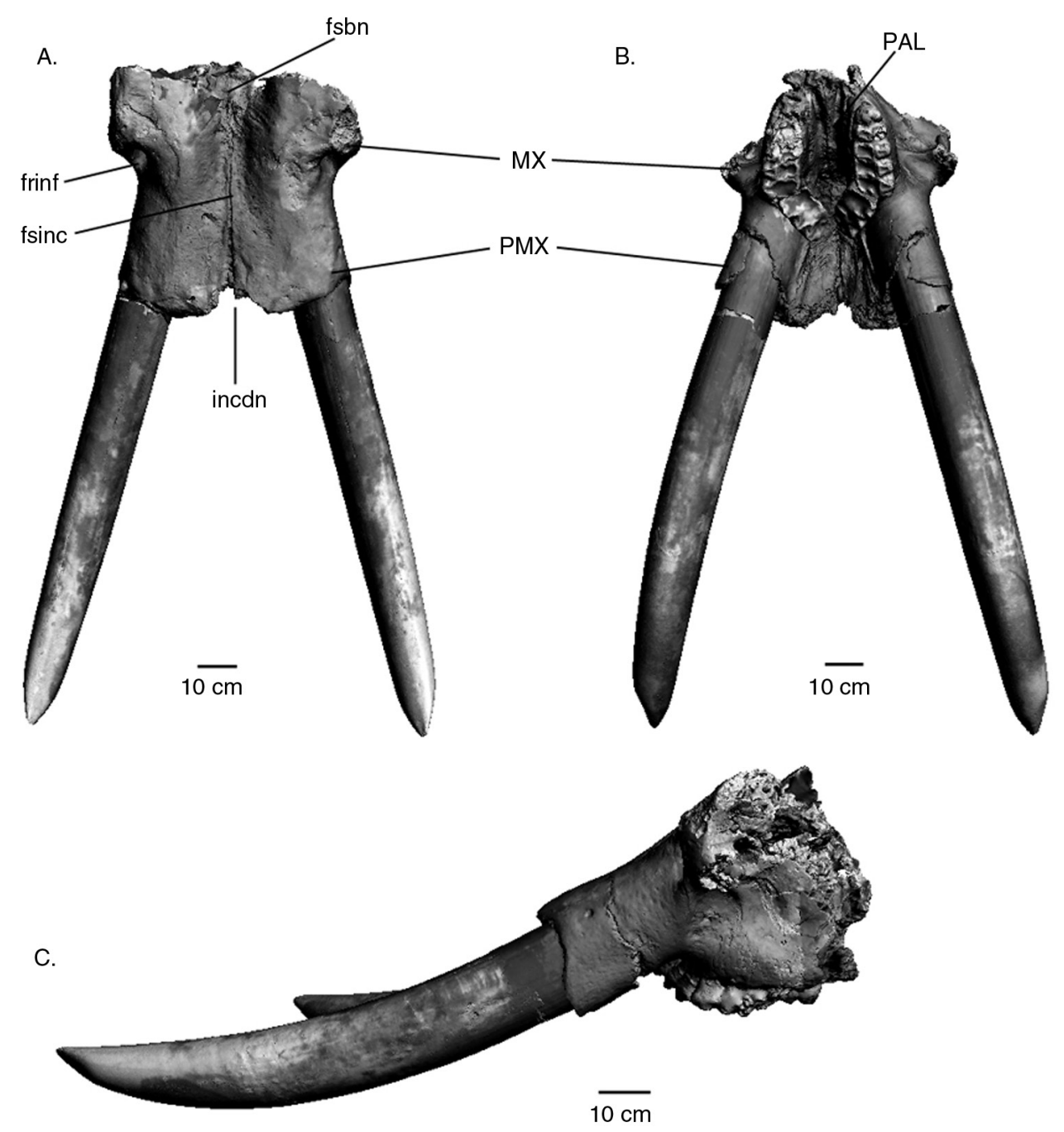

Figura 6.-Cráneo de Stegomastodon platensis de El Trebal 1. A. Vista dorsal; B. vista ventral; C. vista lateral. PMX: Premaxilar; MX: maxilar; PAL: Palatino; fsbn: fosa subnasal; frinf: foramen infraoribitario; fsinc: fosa incisiva; incdn: incisura dental. 
R. Labarca et al.

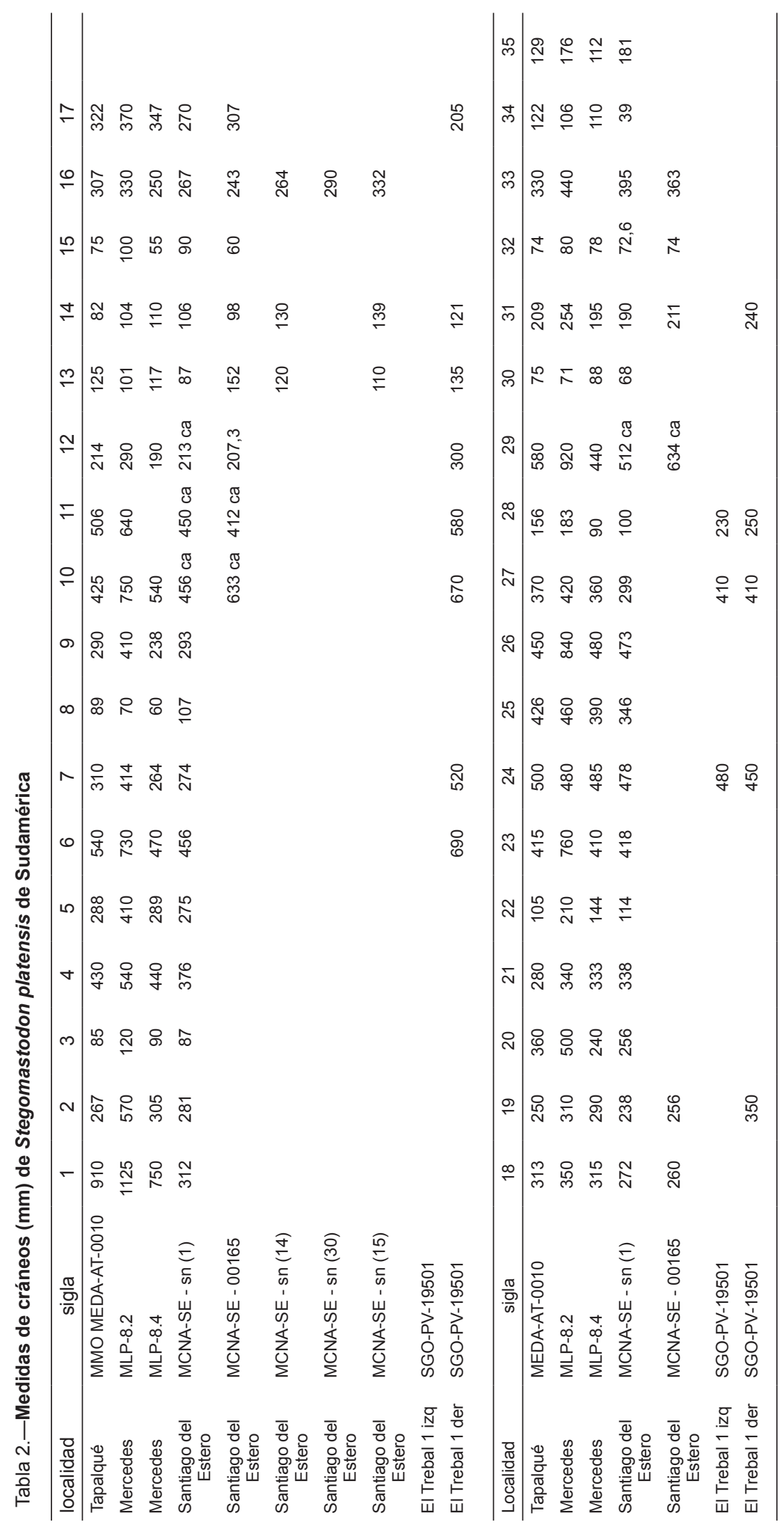


Discusión. Los rasgos morfológicos que permiten la asignación del espécimen al género Stegomastodon son los siguientes: (1) cráneo con la parte anterior de la sínfisis con los premaxilares paralelos, (2) defensas bastante rectas y sin ningún tipo de torsión ni banda de esmalte, ligeramente curvadas en su ápice, (3) fosa incisiva poco desarrollada (Alberdi et al., 2002; Mothé \& Avilla, 2015; Prado et al., 2005).

Los resultados de los análisis bivariantes sitúan los restos dentarios de El Trebal 1 entre los más grandes de Stegomastodon platensis de Argentina (Figura 7). No obstante, y como se ha indicado en trabajos previos (Alberdi et al., 2002, 2008; entre otros), los dientes de los gonfoterios son muy variables en tamaño y morfología aunque mantienen patrones morfológicos generales. Como se puede ver en la Figura 7, los M2/M3 presentan una gran dispersión de tamaños, observando en los diagramas un solapamiento amplio entre las dos especies de Gomphotheriidae incluidas: Cuvieronius hyodon y Stegomastodon platensis de Brasil, Argentina y Chile. Por su parte, los diagramas de caja con los índices de robustez de los molares (Osborn 1936), utilizando como comparación datos de otras localidades de América del Sur, sitúan a los M2 con los restos correspondientes a Stegomastodon platensis que proceden de Chile y Argentina, y con
Cuvieronius hyodon de Bolivia. Por su parte, los de M3 quedarían más próximos a los de Cuvieronius hyodon de Bolivia, si bien los resultados no son concluyentes (Figura 8). Los análisis multivariantes (PCA) realizados con los molares de estas especies resultan igualmente poco significativos ya que los restos de las distintas especies quedan ampliamente solapados (no se incluyen los diagramas por no ser indicativos). Las dimensiones que se han podido obtener del cráneo (Tabla 2) indican que estamos ante un espécimen de tamaño bastante grande, similar al de Mercedes (MLP-8.2, Argentina), asignado a Stegomastodon platensis, uno de los más grandes de los estudiados por nosotros.

Si consideramos que el género Stegomastodon presenta una sola especie válida para Sudamérica (Mothé et al., 2012; Mothé \& Avilla, 2015; Recabarren et al., 2014), S. platensis, los materiales de El Trebal 1 son asignados a esta especie sobre la base de los atributos morfológicos y métricos.

\section{Discusión y conclusiones}

En América del Sur, los primeros restos fósiles con caracteres diagnósticos asignados a la familia Gomphotheriidae se registran en Tarija en torno a 0,99 - 0,76 Ma durante el Pleistoceno medio (Ensenadense) relacionado con el GABI 3 (MacFadden et al., 2013).
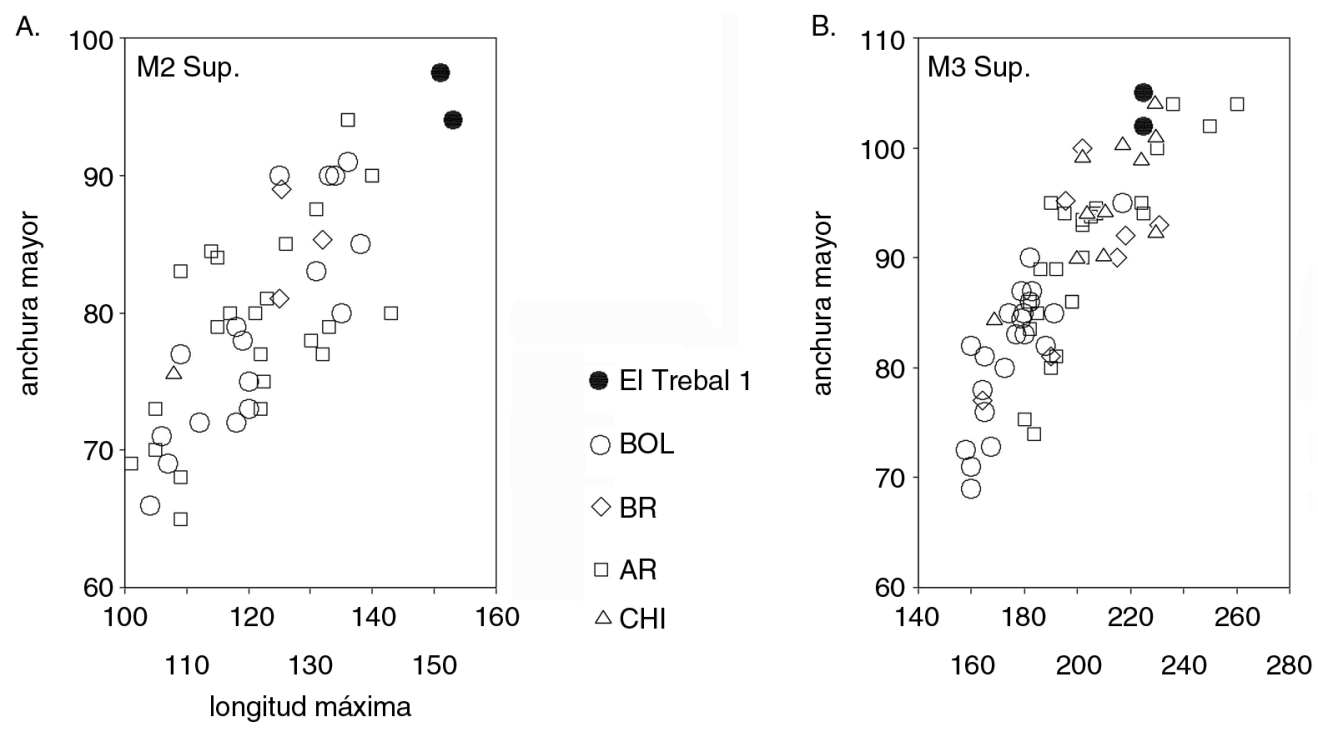

Figura 7.-Diagramas bivariantes $(\mathrm{mm})$ de molares superiores de gonfoterios de América del sur. BOL: Bolivia; BR: Brasil; AR: Argentina; $\mathrm{CHI}$ : Chile. A. M2; B.M3. 

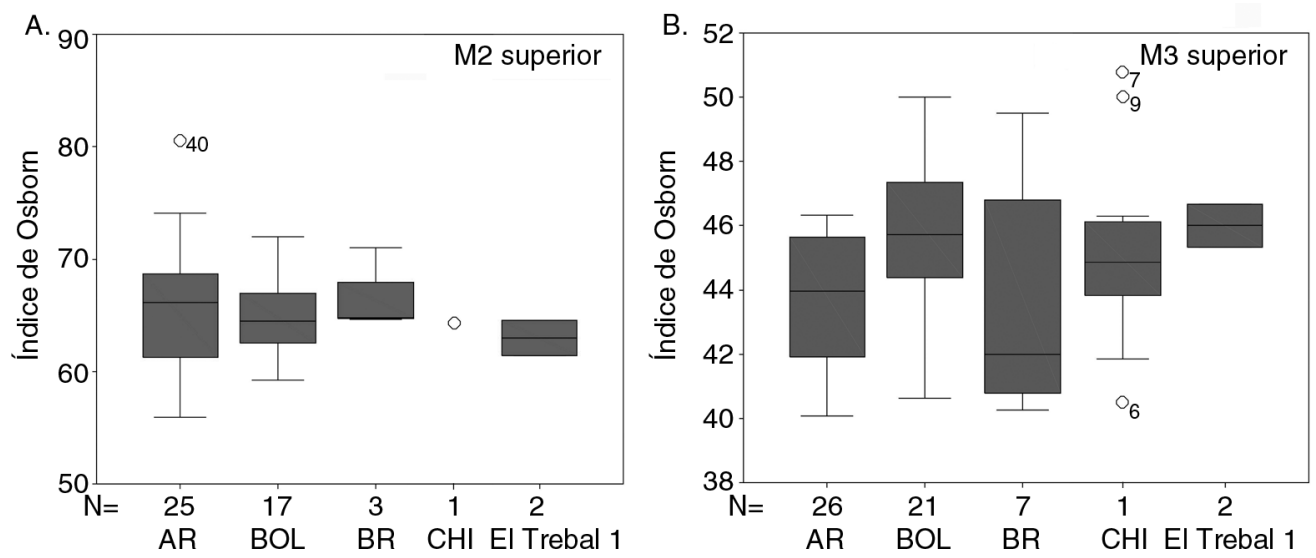

Figura 8.-Diagramas de caja con el índice de robustez de Osborn (1936). A. M2 Superior; B. M3 Superior. Abreviaturas como en Figura 7

López et al. (2001) indican la presencia de un gonfoterio en el Uquiense de Uquía (en torno a 2,5 Ma.) basado en los fragmentos de una vértebra que se debe tomar con precaución. Esta familia pervive en América del Sur hasta el Holoceno temprano y se reconocen dos géneros, Cuvieronius y Stegomastodon (Alberdi et al., 2002, 2007; López et al., 2001; Prado et al., 2002, 2005). Alternativamente, Mothé et al. $(2012,2013)$ y Lucas (Lucas et al., 2011; Lucas \& Alvarado, 2010; Lucas, 2013) postulan que Stegomastodon nunca ingresó a Sudamérica, por lo que los restos asignados a este género deben ser incluidos en Notiomastodon, endémico de Sudamérica.

La aceptación de uno u otro postulado implica considerar dos alternativas evolutivas y biogeográficas, ya que en el primer esquema serían dos los géneros que habrían colonizado Sudamérica en momentos diferentes, mientras que en el segundo caso, $C$. hyodon habría dado origen a $N$. platensis en el subcontinente (Lucas, 2013). Esta discusión descansa, en tres supuestos: (1) la ausencia de restos de Stegomastodon en Centroamérica de acuerdo a la revisión de Lucas \& Alvarado (2010), aun cuando la presencia de Stegomastodon sp. en el sur de Norteamérica fue confirmada en el Pleistoceno Superior de Chapala, México, datados por ${ }^{14} \mathrm{C}$ AMS en 27,910 \pm 270 AP (Alberdi et al., 2009). Éstos fueron posteriormente reasignados a Stegomastodon primitivus Osborn 1936 (Lucas et al., 2011), una especie de la edad Blancano (Plioceno) de América del Norte; (2) las diferencias estratigráficas entre los registros norteamericanos y sudamericanos, ya que Webb (1992) y Lucas et al. (2011), entre otros, señalan que Stegomastodon se registra en América del Norte en las edades Blancano e Irvingtoniano (Plioceno - Pleistoceno Medio), en tanto que las formas sudamericanas serían características de la edad Rancholabreano (SALMA Lujanense, Pleistoceno Superior); y (3) las supuestas diferencias morfológicas entre Notiomastodon y Stegomastodon (p.e. Ferretti, 2010; Lucas, 2008; Lucas et al., 2011; Mothé et al., 2012; Mothé \& Avilla, 2015), aún cuando la osteología de éste último en norteamérica es poco conocida (Lucas et al., 2011). Lucas et al. (2011) han sugerido diferencias postcraneales entre el Stegomastodon primitivus de México y los restos Argentinos de Stegomastodon las que a su juicio serían indicadores de unas extremidades más graviportadoras del primero. Recientemente, Mothé \& Avilla (2015) postularon, a partir de un estudio de morfometría geométrica, diferencias entre la mandíbula de Stegomastodon de Norteamérica y los gonfoterios sudamericanos (Notiomastodon y Cuvieronius de acuerdo a su arreglo). Estos autores apuntaron, además, que la mayor presencia de colinas en Stegomastodon en comparación a las formas sudamericanas permitiría separar ambos grupos. A nuestro juicio, estas inferencias descansan en muy pocos ejemplares como para alcanzar resultados concluyentes, ya que el registro fósil de Stegomastodon en Norteamérica es aún muy escaso y fragmentario (Lucas et al., 2011; Mothé \& Avilla, 
2015). Al respecto, Mothé et al. (2012) han sugerido una importante polimorfía en los gonfoterios de Sudamérica, por lo que es esperable que una situación similar ocurra con otras formas de la familia, como por ejemplo aquellas registradas en Norteamérica.

De esta manera, en este trabajo seguimos la propuesta de Alberdi et al. (2002), que consideran Notiomastodon un sinónimo de Stegomastodon. La convergencia evolutiva entre Stegomastodon de Norteamérica y Notiomastodon de Sudamérica sugerida por Lucas (2013) se explica de mejor manera al considerar a Notiomastodon como sinónimo de Stegomastodon. Creemos que se requiere de un número más significativo de materiales de Stegomastodon de Norteamérica para evaluar eventuales diferencias morfológicas entre éste y las formas sudamericanas.

La historia de la taxonomía de los gonfoterios Chilenos es extensa y se origina con la fundación misma de las dos formas actualmente válidas para Sudamérica (Cabrera, 1929). Hacia finales del siglo pasado, Casamiquela $(1972,1999)$ postuló la presencia de Cuvieronius para el extremo norte ( $c a$. $\left.18^{\circ} 50^{\prime}-26^{\circ} \mathrm{S}\right)$ y Stegomastodon para el centro sur $\left(26^{\circ}-42^{\circ} \mathrm{S}\right)$. Sin embargo, no existe en la actualidad resto fósil alguno proveniente del norte de Chile que tenga valor diagnóstico, tratándose casi en su totalidad de antiguas referencias bibliográficas difíciles de contrastar. Recientemente, López et al. (2010), señalaron la presencia de una costilla de Gomphotheriidae proveniente de Río Salado ( $c a .22^{\circ} 30^{\prime} \mathrm{S}$ ), lo que confirmaría la presencia de gonfoterios en el norte de Chile. Si bien se trata de una unidad anatómica muy poco diagnóstica por lo que esta asignación puede ser puesta en duda, el área debe ser estudiada en detalle debido a su cercanía geográfica con los yacimientos paleontológicos pleistocenos sur bolivianos. La identificación de S. platensis en El Trebal 1 confirma las impresiones de Frassinetti \& Alberdi (2005), Labarca \& Alberdi (2011) y Recabarren et al. (2014) en el sentido de que se trataría de la única forma de gonfoterio que habitó el centro sur de Chile. Cronológicamente, y aunque los materiales no pudieron ser directamente fechados por falta de colágeno, son asignados estratigráficamente al Pleistoceno final - Holoceno temprano (SALMAS Lujanense - Platense). Restos de gonfoterios en localidades cercanas (p.e. Taguatagua, $c a .90 \mathrm{~km}$ al sur de El Trebal 1) presentan fechados radiocarbónicos contextuales coherentes con esta asignación (ca. 13.935-10.761 cal AP, Montané, 1968; Núñez et al., 1994).

Para el tramo final del Pleistoceno, la información polínica proveniente de la cercana localidad de Taguatagua, señala mayor humedad en momentos anteriores a 43.500 cal AP y entre los 40.000 y 21.500 cal AP, con un paisaje dominado por taxa propias del bosque lluvioso Valdiviano (tipo Nothofagus domeyi y Prumnopitys anidna), lo que indica condiciones más lluviosas que las actuales. Dos abruptos períodos de mayor aridez se registran entre los 21000-19500 cal. AP y 17000- 15000 cal. $\mathrm{AP}$, seguidos por nuevos intervalos húmedos entre 19500-17000 cal. AP (coincidente con el Último Máximo Glacial) y 13500-11500 cal. AP. Posterior a este período decrecen las taxas arbóreas drásticamente, siendo reemplazadas por especies propias de ambientes comparativamente más secos (familias Chenopodiaceae y Amaranthaceae), marcando el inicio del Holoceno (Heusser, 1990; ValeroGarcés et al., 2005). Las condiciones húmedas propias del tardiglacial de Chile central sin duda favorecieron la proliferación de grandes herbívoros como los gonfoterios, con dietas $\mathrm{C}_{3}$ (hojas y frutos entre otros), inferidas a partir de isótopos estables (Sánchez et al., 2004).

\section{AGRADECIMIENTOS}

Esta investigación fue financiada por Suez Environnement. Agradecemos a Alexis Dejoux (DIGITAGE) por la digitalización del cráneo del El Trebal 1. Parte de este trabajo se ha realizado en el marco de los Proyectos CGL2010-19116/BOS de la DGCYT, España. Agradecemos a los evaluadores de este trabajo, quienes colaboraron en mejorar el manuscrito.

\section{Referencias}

Alberdi, M.T. \& Prado, J.L. (2008). Presencia de Stegomastodon (Gomphotheriidae, Proboscidea) en el Pleistoceno Superior de la zona costera de Santa Clara del Mar (Argentina). Estudios Geológicos, 64 (2): 175-185. http://dx.doi.org/10.3989/egeol. 08642.044

Alberdi, M.T.; Prado, J.L. \& Cartelle, C. (2002). El registro de Stegomastodon (Mammalia, Gomphotheriidae) en el Pleistoceno superior de Brasil. Revista Española de Paleontología, 17 (2): 217-235. 
Alberdi, M.T.; Prado, J.L. \& Salas, R. (2004). The Pleistocene Gomphotheriidae (Proboscidea) from Peru. Neues Jahrbuch für Geologie und Paläontologie Abhandlungen, 231 (3): 423-452.

Alberdi, M.T.; Prado, J.L.; Ortiz-Jaureguizar, E.; Posadas, P. \& Donato, M. (2007). Historical biogeography of trilophodont gomphotheres (Mammalia, Proboscidea) reconstructed applying dispersion-vicariance analysis. En: $4^{\text {th }}$ European Meeting on the Palaeontology and Stratigraphy of Latin America. Cuadernos del Museo Geominero, 8: 9-14.

Alberdi, M.T.; Cerdeño, E. \& Prado, J.L. (2008). Stegomastodon platensis (Proboscidea, Gomphotheriidae) en el Pleistoceno de Santiago del Estero, Argentina. Ameghiniana, 45 (2): 257-271.

Alberdi, M.T.; Juarez-Woo, J.; Polaco, O.J. \& ArroyoCabrales, J. (2009). Description of the most complete skeleton of Stegomastodon (Mammalia, Gomphotheriidae) recorded for the Mexican Late Pleistocene. Neues Jahrbuch für Geologie und Paläontologie Abhalungen, 251 (2): 239-255.

Cabrera, A. (1929). Una revisión de los mastodontes Argentinos. Revista Museo de La Plata, 32: 61-144.

Campbell, K.E. Jr.; Frailey, C.D.; Romero-Pittman, L. (2000). The late Miocene gomphothere Amahuacatherium peruvium (Proboscidea: Gomphotheriidae) from Amazonian Peru: Implications for the great American faunal interchange. INGEMMET, Boletín, Serie D, Estudios Regionales, 23: 1-152.

Casamiquela, R. (1972). Catalogación crítica de algunos vertebrados fósiles chilenos. II Los Mastodontes. Ameghiniana, 9: 193-208.

Casamiquela, R. (1999). The Pleistocene vertebrate record of Chile. Quaternary of South America and Antarctic Peninsula, 7: 91-107.

Ferretti, M.P. (2010). Anatomy of Haplomastodon chimborazi (Mammalia, Proboscidea) from the late Pleistocene of Ecuador and its bearing on the phylogeny and systematics of South American gomphotheres. Geodiversitas, 32 (4): 663-721. http://dx.doi. org/10.5252/g2010n4a3

Frassinetti, D. \& Alberdi, M.T. (2000). Revisión y estudio de los restos fósiles de mastodontes de Chile (Gomphotheriidae): Cuvieronius hyodon, Pleistoceno Superior. Estudios Geologicos, 56 (3-4): 197-208. http://dx.doi.org/10.3989/egeol.00563-4151

Frassinetti, D. \& Alberdi, M.T. (2005). Presencia del género Stegomastodon entre los restos fósiles de mastodonte de Chile (Gomphotheriidae), Pleistoceno superior. Estudios Geológicos, 61 (1-2): 101-107. http://dx.doi.org/10.3989/egeol.05611-245

Heusser, C. (1990). Ice age vegetation and climate of subtropical Chile. Palaeogeography, Palaeoclimatology, Palaeoecology, 80 (2): 107-127. http://dx.doi.org/ 10.1016/0031-0182(90)90124-P
Labarca, R. \& Alberdi, M.T. (2011). An updated taxonomic view on the family Gomphotheriidae (Proboscidea) in the final Pleistocene of south-central Chile. Neues Jahrbuch für Geologie und Paläontologie Abhandlungen, 262 (1): 43-57. http://dx.doi. org/10.1127/0077-7749/2011/0184

López, G.; Reguero, M.; Lizuain, A. (2001). El registro más antiguo de mastodontes (Plioceno tardío) de América del Sur. Ameghiniana, 38 (S): 35R-36R.

López, P.; Rojas, O.; Mansilla, P.; Olivares, L. \& Martínez, I. (2010). Mamíferos extintos del Pleistoceno de la Cuenca de Calama (Segunda Región, Chile): Nuevos registros y viejas colecciones. Treballs de Museu de Geologia de Barcelona, 17: 11-25.

Lucas, S.G. (2013). The palaeobiogeography of South American Gomphotheres. Journal of Paleogeography, 2 (1): 19-40. http://dx.doi.org/10.3724/ SP.J.1261.2013.00015

Lucas, S.G. \& Alvarado G.E. (2010). Fossil Proboscidea from the upper Cenozoic of Central America: taxonomy, evolutionary an paleobiogeographic significance. Revista Geológica de América Central, 42: 9-42.

Lucas, S.G.; Aguilar, R.H. \& Spielmann, J.A. (2011). Stegomastodon (Mammalia, Proboscidea) from the Pliocene of Jalisco, Mexico and the species-level taxonomy of Stegomastodon. New Mexico Museum of Natural History and Science Bulletin, 53: 517-553.

McFadden, B.J.; Zeitler, P.K.; Anaya, F. \& Cottle, J.M. (2013). Middle Pleistocene age of the fossiliferous sedimentary sequence from Tarija, Bolivia. Quaternary Research, 79 (2): 268-273. http://dx.doi. org/10.1016/j.yqres.2012.12.009

Montané, J. (1968). Primera fecha radiocarbónica de Tagua-Tagua. Noticiario Mensual Museo Nacional de Historia Natural, Chile, 139: 11.

Mothé, D.; Avilla, L.; Cozzuol, M.A. \& Winck, G.R. (2012). Taxonomic revision of the Quaternary gomphotheres (Mammalia: Proboscidea: Gomphotheriidae) from the South American lowlands. Quaternary International, 276-277: 2-7. http://dx.doi. org/10.1016/j.quaint.2011.05.018

Mothé, D.; Avilla, L. \& Cozzuol, M.A. (2013). The South American gomphotheres (Mammalia, Proboscidea, Gomphotheriidae): Taxonomy, phylogeny, and biogeography. Journal of Mammalian Evolution, 20 (1): 23-32. http://dx.doi.org/10.1007/s10914012-9192-3

Mothé, D. \& Avilla, L. (2015). Mythbusting evolutionary issues on South American Gomphotheriidae (Mammalia: Proboscidea). Quaternary Science Review, 110: 23-35. http://dx.doi.org/10.1016/j. quascirev.2014.12.013

Núñez, L.; Varela, J.; Casamiquela, R.; Schiappacasse, V.; Niemeyer, H. \& Villagrán, C. (1994). Cuenca de 
Taguatagua en Chile: el ambiente del Pleistoceno Superior y ocupaciones humanas. Revista Chilena de Historia Natural, 67: 503-519.

Osborn, H.F. (1936). Proboscidea. A monograph of the mastodons and elephants. Vol 1-Moeritherioidea, Deinotheiroidea, Mastodontoidea. Bulletin of the American Museum of Natural History, 1: 1-802.

Prado, J.L. \& Alberdi, M.T. (2011). Équidos y Gonfoterios del Pleistoceno tardío de San Pedro, provincia de Buenos Aires, Argentina. Estudios Geológicos, 68 (2): 261-276. http://dx.doi.org/10.3989/egeol.40422.143

Prado, J. L.; Alberdi, M. T. \& Gómez, G. (2002). Late Pleistocene gomphotheres (Proboscidea) from the Arroyo Tapalque locality (Buenos Aires, Argentina) and their taxonomic and biogeographic implications. Neues Jahrbuch für Paläontologie und Geologie Abhandlungen, 225 (2): 275-296. http://dx.doi. org/10.1127/njgpa/225/2002/275

Prado, J.L.; Alberdi, M.T.; Azanza, B.; Sánchez, B. \& Frassinetti, D. (2005). The Pleistocene Gomphotheriidae (Proboscidea) from South America. Quaternary International, 126-128: 21-30. http://dx.doi. org/10.1016/j.quaint.2004.04.012

Prothero, D.R.; Davis, E.B.; Hopkins, S.S.B. (2008). Magnetic stratigraphy of the Massacre Lake Beds (late Hemingfordian, Miocene), northwest Nevada, and the age of the "proboscidean datum" in North America. New Mexico Museum of Natural History and Science Bulletin, 44: 239-246.

Recabarren, O.P.; Alberdi, M.T. \& Pino, M. (2014). La Familia Gomphotheriidae en América del Sur: evidencia de molares al norte de la Patagonia chilena. Estudios Geológicos, 70 (1): e001. http://dx.doi. org/10.3989/egeol.41346.273
Reguero, M.A.; Candela, A.M. \& Alonso, R.N. (2007). Biochronology and biostratigraphy of the Uquia Formation (Pliocene-early Pleistocene, NW Argentina) and its significance in the great American biotic interchange. Journal of South American Earth Sciences, 23 (1): 1-16. http://dx.doi.org/10.1016/j. jsames.2006.09.005

Sánchez, B.; Prado, J.L. \& Alberdi, M.T. (2004). Feeding ecology, dispersal, and extinction of South American Pleistocene gomphotheres (Gomphotheriidae, Proboscidea). Paleobiology, 30 (1): 146-161. http:// dx.doi.org/10.1666/0094-8373

Sellés, D. \& Gana, P. (2001). Geología del área TalaganteSan Francisco de Mostazal. Regiones de Metropolitana de Santiago y del Libertador General Bernardo O’Higgins. Servicio Nacional de Geología y Minería. Carta Geológica de Chile, Serie Geología Básica No. 74, 30 p. 1 mapa escala 1:100.000, 1 anexo, Santiago.

Valero-Garcés, B.; Jenny, B.; Rondanelli, M.; Delgado-Huertas, A.; Burns, S.; Veit, H. \& Moreno, A. (2005). Palaeohydrology of Laguna de Tagua Tagua $\left(34^{\circ} 30^{\prime} \mathrm{S}\right)$ and moisture fluctuations in Central Chile for the last $46000 \mathrm{yr}$. Journal of Quaternary Science, 20 (7-8): 625-641. http://dx.doi.org/10.1002/jqs.988

Varela, J. (1991). Geología del Cuaternario de la Depresión Central de Chile en la Zona de la Cuenca de Santiago, región Metropolitana, Chile. En: Congreso Geológico Chileno, 6, Resúmenes expandidos: 593-596.

Webb, S.D. (1992). A brief history of New World Proboscidea with emphasis on their adaptations and interactions with man. En: Fox, J. W.; Smith, C. B. \& Wilkins, K. T. (Eds). Proboscidean and Paleoindian Interactions. Baylor University Press, 16-34. 